Revista de Psicología de la PUCP. Vol. XIII. No 2. 1995

\title{
EVALUACION DEL CLIMA MOTIVACIONAL DE CLASE
}

\author{
Luisa Irureta Núñez
}

En el presente estudio, se adaptó y validó para la población venezolana el cuestionario Clima Motivacional de Clase -CMC-1- desarrollado en España por Alonso y García (1987) para alumnos cursantes de la tercera etapa de EGB -11 a 15 años-. Constituyeron la muestra 1690 alumnos, de ambos sexos, cursantes la tercera etapa de Educación Básica $-7^{\circ}, 8^{\circ}$ y $9^{\circ}$ grados- y procedentes de 12 escuelas de Caracas -6 públicas y 6 privadas-. El análisis factorial detectó una estructura de 6 factores diferentes a la derectada en el estudio español.

Palabras Claves: clima social de la clase, interacciones en el aula, cuestionario CMC -1 , Venezuela, análisis factorial, validez predictiva.

\section{Assessment of the class motitotional climate}

This study adapted and validated the CMC-1 questionnaire on Class Motivational Climate to the Venezuelan population. The questionnaire was developed in Spain by Alosno and Garcia (1987) for students aged 11 to 15 . The sample contained 1,690 students of both sexes in 7 th, 8 th, and 9 th grades from 12 schools in Caracas (six public and six private). The factorial analysis detected a structure of six factors different from the one detected in the Spanish study.

Keywords: social climate of the class, classroom interactions, CMC-1 questionnaire, Venezuela, factor analysis, predictive validity, school elementary education.

1 Personal docente y de investigación de la Universidad Central de Venezuela en la categoría de Asociada. Docente en la Escuela de Psicología y el Post-grado en psicología de la Instrucción. Proyecros de investigaión en el área de Psicología Educativa, orientados al estudio de factores psicosociales de los docentes y estudiantes que afectan la motivación por las actividades académicas y por el aprendizaje. Doctora en Psicología de la Universidad Autónoma de Madrid, Magister Scientiarum en Educación y Licenciada en Psicología de la Universidad Central de Venezuela. Dirección Postal Apartado Postal 63463. Código postal 1067 A. Caracas-Venczuela Teléfonos: (582) 7826042 Fax: (582) 79417 14. Correo-E. LIRURETA@DINO.CONICIT.VE Y LIRURETA@SAGI.UCV.EDU.VE 



\section{Introducción}

Si los profesores influyen en la motivación de sus alumnos, está claro que lo hacen a través del clima motivacional que crean en clase con su forma de presentar las tareas, con el tiempo y la atención que dedican a cada alumno individualmente, con los mensajes que dan a lo largo de la actividad escolar antes, durante y después de la realización de las tareas que deben hacer los alumnos, con el tipo de interacción que promueven entre éstos, etc. Por este motivo parece importante conocer no sólo lo que los profesores piensan que deben hacer para motivar a sus alumnos o las actitudes y expectativas que pueden tener sobre la posibilidad de conseguirlo sino, además, qué clima motivacional crean en sus clases.

Contar con instrumentos para evaluar el clima de clase tendría evidentes repercusiones prácticas. Por una parte, servirían para el diagnóstico del grado de adecuación de las prácticas docentes en cuanto, al menos parcialmente, a la percepción de los alumnos del ambiente en el que se desarrollan sus clases. Por otra parte, podrían proporcionar información específica para determinar qué tipos de ayuda requieren los profesores que los capacite para crear en las aulas ambientes adecuados para el proceso enseñanza aprendizaje. Finalmente, podrían servir como indicador del cambio que se produce en las clases como resultado de programas de ayuda al profesorado.

Como plantea Alonso (1992), evaluar el clima motivacional de la clase implica responder a una serie de preguntas relacionadas tales como: ¿Cómo se organiza de hecho la actividad en las aulas? ¿Esa organización responde básicamente a uno u otro de los distintos sistemas motivacionales descritos en la literatura -individual, cooperativo, competitivo-? ¿Existe alguna dimensión característica de la organización de la actividad que, sin estar específicamente ligada al tipo de interacción que se dé en el aula, afecte 
a la motivación en general -el ritmo de trabajo, la claridad o no de los objetivos a conseguir y de los procedimientos a seguir, el grado de alboroto y los tiempos sin tener que hacer, las preferencias mostradas por el profesor, etc.-? ¿Cuál es papel del profesor en todo esto? Para dar respuestas a tales preguntas parece viable preguntar a los alumnos, hecho que ha dado lugar a la construcción de diferentes cuestionarios de clima de clase.

Para dar respuesta a estas preguntas también es preciso partir de algún modelo que sugiera qué pautas de acción pueden ser relevantes. Trabajos anteriores han puesto de manifiesto la necesidad de tener en cuenta variables como la atención individualizada, el ritmo de la clase, la claridad de objetivos, el orden (Anderson, 1973; Silbergeld y otros, 1977, 1979; Owens y Straton, 1980), los mensajes que da el profesor antes, durante y después de las tareas (Alonso y Sola, 1987) y el sistema de interacciones que promueve entre los alumnos (Johnson y Johnson, 1985), entre otras. Por ejemplo, la organización de la actividad escolar en grupos cooperativos parece ser, bajo ciertas condiciones, un factor especialmente útil tanto para estimular el interés y el esfuerzo de los alumnos por aprender como para facilitar el rendimiento de éstos; lo mismo cabe decir de los mensajes que orientan la atención del alumno hacia el proceso de realización de la tarea más que hacia el resultado, clarificando objetivos, mostrando cómo pensar para resolver problemas y buscar la superación de los errores, etc.

Desde estos presupuestos se han desarrollado en España varios cuestionarios de clima motivacional de clase, entre otros el cuestionario CMC1 (Alonso y García, 1987), este cuestionario ha sido adaptado a las peculiaridades del uso (giros y palabras) del idioma español en Venezuela y validado para su uso con población venezolana.

El cuestionario, en su versión española fue construido para alumnos cursantes de la tercera etapa de Educación General Básica -de 11 a 15 años-; consta de 70 elementos formulados en sentido positivo y negativo de forma equilibrada y en él se pide al alumno que identifique las características de la organización de las actividades escolares que pueden tener repercusión en su interés y esfuerzo por el estudio, tales como:

a) Ritmo de la clase. Se manifiesta en aspectos tales como la velocidad con que el profesor explica, el tiempo que da para la realización de las tareas, el tiempo que se detiene en cada tema, etc. Consideramos 
que un ritmo excesivamente rápido puede ser desmotivante al dificultar la comprensión y elaboración de la información, lo que generaría ansiedad en los alumnos. Por otra parte, si es excesivamente lento, puede aburrir a los alumnos.

b) Dificultad percibida de las distintas materias, textos, explicaciones $y$ exámenes. Si es alta, puede generar ansiedad al afectar las expectativas del sujeto de conseguir los objetivos escolares.

c) Orden. Esta característica hace referencia a aspectos tales como la puntualidad con que empiezan las clases, el nivel de ruido y de movimiento, la permisividad de alboroto en clase, etc., aspectos que contribuyen según el grado en que se presenten a crear un clima de trabajo o, por el contrario, de deterioro de la actividad escolar.

d) Grado de especificación de objetivos y actividades. Saber qué se persigue y qué ha de hacerse para alcanzar una meta puede contribuir a que el sujeto se esfuerce. Por el contrario, la ausencia de objetivos claros de aprendizaje o no saber qué se ha de hacer para lograrlos puede crear desorientación y frustración a los alumnos, lo que afecta negativamente al esfuerzo de éstos.

e) Trabajo en grupo. Los elementos incluidos en esta categoría hacen referencia al grado en que se trabaja de hecho en grupo en las clases, a la preferencia por trabajar así, a las conductas de ayuda y a la valoración del trabajo en grupo para el aprendizaje, al hecho de que el profesor evalúe a los alumnos en base a los trabajos colectivos y no sólo en base a los trabajos realizados individualmente, etc., entre otros aspectos.

f) Competición. Esta categoría incluye elementos que hacen referencia a aspectos como el favoritismo del profesor hacia los mejores, al hecho de que compare frecuentemente a los alumnos desde esta perspectiva, a que las actividades se organicen de forma que se ponga de manifiesto al término de las mismas quienes son mejores y peores, etc.

g) Individualismo. Los elementos referidos a esta caregoría evalúan aspectos tales como el hecho de que se trabaje normalmente de modo individual no comperitivo, pero también aspectos tales como que el 
profesor se preocupe por el progreso individual y el grado de apoyo y estímulo que manifieste hacia los progresos del alumno.

Los trabajos realizados en España con el CMC-1 pusieron de manifiesto la existencia de un estructura de seis factores, los que aparecen en el cuadro 1.

Cuadro 1: Escalas originales del cuestionario de clima de clase $\mathrm{CMC}^{-2}$

CMC-1 (EGB)

1. Ritmo de las clases agobiante, con poco tiempo para realizar las tareas VERSUS ritmo adecuado.

2. Favoritismo del profesor hacia los más listos VERSUS equidad en el trato individual a cada alumno.

3. Ambiente de trabajo, preferentemente en grupo, con orden, organización y claridad de objetivos

VERSUS preferencia por el trabajo individual, alboroto, desorganización y confusión respecto a los objetivos a conseguir.

4. Ambiente en que se nota el interés del profesor por que cada alumno aprenda

VERSUS ausencia de dicho interés.

5. Preferencia por la cooperación y el trabajo en grupo

VERSUS rechazo del trabajo en grupo y preferencia por el trabajo individual.

6. Clima competitivo en que cada uno va a lo suyo

VERSUS clima cooperativo y de ayuda.

\section{Adaptación y validación del CMC para población venezolana.}

\section{Metodología}

La adaptación y validación del cuestionario CMC-1 se ha hecho siguiendo una secuencia de pasos a través de los que se ha tratado de responder a las siguientes preguntas: 
- Si se corrigen los cuestionarios contestados por los alumnos de Venezuela de acuerdo con la estructura del cuestionario derivada de los análisis españoles, ¿se mantiene a un nivel semejante la confiabilidad de las distintas escalas del mismo? A esta pregunta responde el análisis de la consistencia interna de las distintas escalas.

- En caso de que la confiabilidad no sea semejante, ¿se debe a que la estructura motivacional de los alumnos de Venezuela, en la medida en que puede ser detectada por el cuestionario, es significativamente distinta a la de los alumnos españoles? A esta pregunta responden los análisis factoriales de primero y segundo orden realizados a partir de los cuestionarios de los alumnos de Venezuela. Estos análisis son especialmente importantes en el caso del CMC-1 porque a los 70 elementos originales se añadieron otros 37 a fin de dar mayor longitud a algunas de las escalas y aumentar de este modo su fiabilidad.

- Con independencia de que la estructura factorial detectada en Venezuela sea más o menos diferente de la detectada en España, ¿es fiable evaluar a los alumnos corrigiendo sus cuestionarios a partir de la estructura identificada en Venezuela? A esta pregunta responde el análisis de la consistencia interna de las distintas escalas identificadas a partir del análisis factorial.

- Finalmente, utilizando como base la estructura factorial que haya resultado más confrable, ¿cuál es la capacidad predictiva de las escalas del cuestionario en relación con los criterios de rendimiento?

Como paso previo a la aplicación de la prueba, los 70 ítemes originales fueron matizados para adaptar su formulación a las peculiaridades (giros y palabras) del uso del idioma español en Venezuela; además se diseñaron especialmente para esta versión 37 ítems adicionales.

\section{Sujetos}

Los sujetos fueron 1690 alumnos, niños y niñas, cursantes de la tercera etapa de Eucación Básica $-7^{\circ}, 8^{\circ}$ y $9^{\circ}$ grados-, procedentes de 6 escuelas públicas y 6 privadas del área metropolitana de Caracas. 


\section{Resultados y Discusión}

\section{Consistencia interna de las escalas originales}

En la tabla 1, además de la descripción de las puntuaciones obtenidas por los sujetos en las distintas escalas del cuestionario, puntuaciones derivadas de acuerdo con los criterios establecidos en España (Alonso, 1992), se incluyen los coeficientes de consistencia interna $(\alpha)$ originales junto con los obtenidos en Venezuela. En este primer análisis sólo se utilizaron los 70 elementos de los que consta la prueba española. Como puede comprobarse, son muy semejantes, excepto en el caso de los factores 5 y 6 que son más bajos. Este hecho muestra que la mayoría de los factores identificados en España tienen cierta validez transcultural. No obstante, dada la baja confiabilidad de los dos últimos factores, es necesario proceder a un análisis factorial de las respuestas de los sujetos al cuestionario completo de 107 elementos.

\section{Tabla 1: Cuestionario CMC}

\begin{tabular}{|cccccc|}
\hline \multicolumn{5}{|c|}{ CARACTERISTICAS Y CONSISTENCLA INTERNA. } \\
$\begin{array}{r}\text { Comparación de la consistencia interna del estudio español } \\
\text { con la de los datos venezolanos corregidos a partir de la } \\
\text { estructura identificada en España }\end{array}$ \\
\hline Factores & Sujetos & Media & Sx & $\alpha$ & $\begin{array}{c}\alpha \text { en } \\
\text { España }\end{array}$ \\
\hline F1 & 1687 & 44.71 & 17.84 & 0.86 & 0.91 \\
F2 & 1690 & 11.26 & 6.40 & 0.76 & 0.85 \\
F3 & 1690 & 18.04 & 6.09 & 0.60 & 0.78 \\
F4 & 1687 & 31.36 & 7.25 & 0.62 & 0.68 \\
F5 & 1690 & 20.98 & 4.50 & 0.42 & 0.68 \\
F6 & 1690 & 10.77 & 4.09 & 0.44 & 0.52 \\
\hline
\end{tabular}




\section{Análisis factoriales}

\section{A) Análisis factorial de primer orden}

Las respuestas de los alumnos se han analizado factorialmente utilizado el método de Componentes Principales para la obtención de factores y el método Oblimín para su rotación. El criterio de inclusión de un elemento en un factor ha sido que su saturación fuese superior a $=.30$. En el caso de elementos que cumplen con el criterio de inclusión para más de un factor, han sido incluidos sólo en el factor en el que su saturación es mayor.

\section{Tabla 2: Factores}

\begin{tabular}{|l|}
\multicolumn{1}{|c|}{$\begin{array}{c}\text { Escalas del cuestionario. Clima de clase ampliado. } \\
\text { Adaptación venezolana (CMC-VENZ) }\end{array}$} \\
\hline FACTORES DE PRIMER ORDEN \\
\hline 1. $\begin{array}{l}\text { Clima de interés porque el alumno aprenda, poniendo para ello todos } \\
\text { los medios necesarios VERSUS todo lo contrario. } \\
\text { (No coincide con ninguno de los factores de la escala original, se aproxi- } \\
\text { ma al 4to factor de la versión original) }\end{array}$ \\
2. $\begin{array}{l}\text { Ritmo agobiante, con poco tiempo para realizar las tareas VERSUS ritmo } \\
\text { adecuado. } \\
\text { (Coincide totalmente con el primer factor de la versión original) }\end{array}$ \\
3. $\begin{array}{l}\text { Favoritismo del profesor hacia los mejores VERSUS equidad en el trato } \\
\text { individual a cada alumno. } \\
\text { (Coincide en su significado con el segundo factor de la versión original) }\end{array}$ \\
4. Rechazo por el trabajo en grupo, insolidaridad y preferencia por el trabajo \\
individual VERSUS todo lo contrario \\
(No coincide con ninguno de los factores de la escala original, su sig- \\
nificado se aproxima en opuesto al del quinto factor de la versión original) \\
5. Alboroto y desorganización VERSUS orden, organización y claridad de \\
objetivos. \\
(Su significado es opuesto al del tercer factor de la versión original) \\
6. Trabajo en grupo de hecho VERSUS ausencia de esta forma de trabajo. \\
(No coincide con ninguno de los factores de la escala original)
\end{tabular}


El análisis de los resultados, que se recogen en la tabla 2, pone de manifiesto que la estructura factorial es parcialmente diferente a la identificada en España. Aparecen seis factores que explican el 30,8 \% de la varianza antes de la rotación, siendo el índice de fiabilidad Theta de Carmines obtenido en base al análisis de 0.95. Las escalas y los elementos que las integran se exponen en la tabla 2 y en el apéndice donde se indica como obtener las puntuaciones en las mismas.

El primer factor incluye un total de 29 elementos, todos menos uno con saturación positiva. No coincide plenamente con ninguno de los factores identificados en los análisis españoles, aunque por los elementos de que se compone su significado se aproxima al del factor 4 . Se trata de un factor o dimensión en el que los alumnos que puntúan alto ven a su profesor con todas las virtudes que son deseables en un profesor: se preocupa porque cada alumno aprenda, sin favoritismo, ayudando al que más lo necesita, llevando para ello un ritmo adecuado, aclarando objetivos, ayudando en las dificultades, tratando de mostrar la relevancia de lo que se estudia, elogiando individualmente a cada uno por sus progresos individuales, orientando más hacia el proceso que hacia el resultado, etc. Por el contrario, puntuar bajo equivaldría a ver al profesor como carente de todas esas virtudes. Puede denominarse, en consecuencia, este factor como el cuarto de los identificados en España: "Clima de interés porque el alumno aprenda, poniendo para ello todos los medios necesarios, versus todo lo contrario".

El segundo factor incluye un total de 21 elementos, 6 de los cuales saturan negativamente. Coincide con el primero de los factores identificados en España, si bien faltan algunos elementos cuya desaparición ha sido compensada por la inclusión de tres de los elementos nuevos creados para la adaptación venezolana. El contenido de los elementos hace referencia al hecho de que el profesor lleva un ritmo demasiado rápido, lo que hace que las clases resulten agobiantes y que a veces los objetivos no queden demasiado claros. En consecuencia, puede seguirse denominando este factor como de "Ritmo agobiante, con poco tiempo para realizar las tareas, versus ritmo adecuado".

El tercer factor incluye 15 elementos, todos menos dos con saturación positiva. Coincide plenamente en su significado -aunque no en el número de elementos- con el segundo de los factores identificados en España. En 202 
la medida en que los alumnos de una clase puntúen alto en este factor nos están diciendo que su profesor no es equitativo, dedicándose a los mejores y despreocupándose de los que tienen dificultades. En consecuencia, puede seguirse denominando como factor de "Favoritismo del profesor hacia los mejores versus equidad".

El cuarto factor no coincide claramente, en lo que a los elementos de que consta se refiere, con ninguno de los identificados en España. Sin embargo, su significado se aproxima al del factor 5 , si bien invirtiendo el sentido de las puntuaciones. Consta de 11 elementos, tres con saturación negativa, que hacen referencia a un clima de individualismo, insolidaridad y preferencia por el trabajo individual versus un clima de cooperación, ayuda y preferencia por el trabajo en grupo. La mayoría de los elementos no hacen referencia al profesor, sino a las relaciones entre compañeros. En consecuencia, puede denominarse este factor como de "Rechazo del trabajo en grupo, insolidaridad y preferencia por el trabajo individual versus todo lo contrario".

El quinto factor coincide básicamente con el factor 3 de los identificados en España. Incluye 9 elementos, cuatro con saturación positiva y cinco con saturación negativa. Su contenido hace referencia al hecho de que en clase hay frecuentes alborotos, impuntualidad, falta de objetivos claros y frecuentes tiempos muertos en los que no se tiene algo que hacer o bien, en el caso de los elementos con saturación negativa, a todo lo contrario. En consecuencia, creemos que puede denominarse este factor como de "alboroto y desorganización versus orden y claridad de objetivos".

Finalmente, el sexto factor incluye 7 elementos, 2 de ellos con saturación negativa. Las puntuaciones altas indican que se percibe que en clase se trabaja de hecho frecuentemente en grupo -con independencia de que esta forma de trabajo sea la preferida de los alumnos o no-, actividad que se ve unida a la percepción de tiempos muertos frecuentes y a un modo de calificar basado en la comparación entre alumnos. Así pues, hemos denominado este factor como "Trabajo en grupo de hecho versus ausencia de esta forma de trabajo".

No saturan en ningún factor 15 elementos, 12 de la versión original y 3 de los diseñados especialmente para la versión venezolana. 


\section{B) Análisis factorial de segundo orden}

La existencia de correlaciones significativas entre los seis factores del CMC-1 ampliado, correlaciones que presentamos en la tabla 3, hizo aconsejable la realización de un análisis de dichas correlaciones (equivalente a un análisis factorial de segundo orden) con el fin de precisar de modo más adecuado las dimensiones del clima motivacional del aula.

Tabla 3: Correlaciones entre las escalas correspondientes a los factores de primer orden del CMC-1.

\begin{tabular}{|ccccccc|}
\hline & F1 & F2 & F3 & F4 & F5 & F6 \\
\hline F1 & 1.00 & & & & & \\
F2 & -0.78 & 1.00 & & & & \\
F3 & -0.66 & 0.71 & 1.00 & & & \\
F4 & -0.19 & 0.23 & 0.28 & 1.00 & & \\
F5 & -0.44 & 0.35 & 0.32 & 0.01 & 1.00 & \\
F6 & 0.10 & -0.05 & 0.02 & -0.58 & 0.22 & 1.00 \\
\hline
\end{tabular}

Para este análisis utilizó el método de Componentes Principales para la extracción de factores y el método Oblimín para la rotación. El criterio de inclusión de una escala en un factor es que la saturación fuese superior a 0.40 . Los resultados de este análisis se presentan en la tabla 4 .

Han aparecido dos factores que se describen en la parte inferior de la tabla 4, que explican el $73 \%$ de la varianza antes de la rotación. En el primero saturan significativamente las escalas correspondientes a los factores de primer orden 1 (con saturación negativa), 2, 3 y 5 factores. Se trata de una dimensión en la que puntuar alto implica percibir al profesor de forma muy negativa, ya que a la falta de preocupación por el alumno se une el favoritismo, el llevar un ritmo agobiante y el permitir el alboroto y el desorden en sus clases, lo contrario de lo que ocurre si la puntuación es baja.

En cuanto al segundo factor de segundo orden, en el que satura negativamente el factor 4 y positivamente el 6 , puntuar alto significa que 204 
se percibe una preferencia por el trabajo en grupo y una actitud solidaria por parte de los compañeros junto al hecho de trabajar efectivamente en grupo de modo frecuente.

Tabla 4: Análisis Factorial de Segundo Orden del cuestionario CMC-1 ampliado. Método de Componentes Principales y rotación Oblimín.

\begin{tabular}{|c|c|c|}
\hline Sujetos: 1673 & \multicolumn{2}{|c|}{ Factores } \\
\hline Elementos & VCMC-I & VCMC-II \\
\hline VCMC-F1 & -0.89 & 0.00 \\
\hline VCMC-F2 & 0.88 & 0.00 \\
\hline VCMC-F3 & 0.83 & 0.00 \\
\hline VCMC-F4 & 0.00 & -0.83 \\
\hline VCMC-F5 & 0.64 & 0.33 \\
\hline VCMC-F6 & 0.00 & 0.90 \\
\hline Valor propio & 2.72 & 1.65 \\
\hline \multicolumn{3}{|c|}{$\begin{array}{l}\text { Escalas del cuestionario. Clima de clase ampliado Adaptación venezolana } \\
\text { (CMC-VENZ) }\end{array}$} \\
\hline \multicolumn{3}{|c|}{$\begin{array}{l}\text { 1. Percibir el profesor en forma muy negativa, por falta de preocupación } \\
\text { por el alumno, favoritismo por los mejores, impone un ritmo agobiante } \\
\text { y permite el alboroto y desorden en clase. } \\
\text { (Factores de primer orden -1 er., 2do., 3ro. y } 5 \text { to) }\end{array}$} \\
\hline \multicolumn{3}{|c|}{$\begin{array}{l}\text { 2. Preferencia por el trabajo en grupo y el que de hecho se trabaja en grupo } \\
\text { frecuentemente. } \\
\text { (Factores de primer orden -4to. y Gto. }\end{array}$} \\
\hline
\end{tabular}

Consistencia interna de las escalas derivadas a partir de datos venezolanos

Los análisis factoriales realizados han puesto de manifiesto que, pese a existir cierta semejanza entre los resultados de los análisis originales y los 
realizados en Venezuela, las escalas derivadas de los mismos no son totalmente idénticas. De hecho, aunque lo fueran en su significado, era casi imposible que lo fueran, dado que en estos análisis había más elementos. Este hecho abre la posibilidad de utilizar las escalas derivadas de los análisis venezolanos a la hora de evaluar el clima motivacional del aula. Sin embargo, es preciso comprobar la consistencia interna de estas escalas y compararla con la de las escalas derivadas a partir de los análisis realizados en España. Sólo en la medida en que dicha consistencia sea mayor sería aconsejable sustituir las escalas originales por las derivadas de los análisis factoriales descritos.

En la tabla 5 se recogen, junto a los estadísticos que describen los datos de las escalas mencionadas, su consistencia interna. Si se comparan estos datos con los recogidos en la tabla 1 puede comprobarse que los índices actuales son algo mayores, lo que era de esperar dada la mayor longitud de las escalas. Este hecho sugiere que, si los datos de validez que analizaremos en breve son adecuados, será preferible utilizar las escalas construidas a partir de los análisis factoriales venezolanos.

Tabla 5: Cuestionario CMC-I ampliado. Características y consistencia interna.Datos venezolanos corregidos a partir de la estructura identificada originalmente en Venezuela

\begin{tabular}{|lccccc|}
\hline \multicolumn{5}{|c}{ Datos de escalas correspondientes a } & \multicolumn{3}{c|}{ Factores de Primer Orden } \\
\hline Factores & Sujetos & Media & Sx & $\alpha$ & $\begin{array}{c}\text { Factor } \\
\text { español } \\
\text { equivalente }\end{array}$ \\
\hline VCMC-F1 & 1684 & 95.79 & 24.26 & 0.92 & CMC 4 ? \\
VCMC-F2 & 1684 & 44.18 & 18.88 & 0.89 & CMC 1 \\
VCMC-F3 & 1687 & 26.25 & 13.28 & 0.87 & CMC 2 \\
VCMC-F4 & 1682 & 24.11 & 9.73 & 0.77 & CMC 5, 6 \\
VCMC-F5 & 1690 & 18.59 & 7.56 & 0.76 & CMC 3 \\
VCMC-F6 & 1690 & 14.04 & 5.79 & 0.63 & - \\
VCMC-FI & 1681 & 141.14 & 54.95 & 0.95 & - \\
VCMC-FII & 1682 & 49.92 & 13.94 & 0.82 & - \\
\hline
\end{tabular}


Validez predictiva

Para estudiar la validez predictiva del CMC-1 se ha utilizado como criterio la nota obtenida por cada alumno en la asignatura correspondiente al profesor en relación con cuyas clases se respondía el cuestionario. En cuanto a los predictores, se han utilizado tres grupos: a) Las puntuaciones correspondientes a las escalas del cuestionario original de 70 elementos; b) Las correspondientes a las seis escalas identificadas en el análisis de las respuestas al cuestionario ampliado a 107 elementos, y c) las correspondientes a las dos escalas derivadas a partir del análisis factorial de segundo orden.

Los resultados se muestran en las tablas 6 a 8 . Como puede comprobarse, el valor de $\mathrm{R}$ es mayor en el caso de corregir la prueba de acuerdo con su estructura original, lo que sorprende un poco debido a la menor confrabilidad de dos de las escalas; sin embargo, si examinamos el peso que cada escala de primer orden tiene en la predicción, podemos ver que en el caso de utilizar la estructura derivada en Venezuela sólo los factores 2,3 y 4 , con una correspondencia bastante marcada con los factores españoles, tienen un peso significativo. Tal vez esto pueda deberse a que el primer factor puede encerrar una apreciación maniquea del profesor es bueno o es malo- de tipo global que no responde a lo que en realidad ocurre en la clase. No obstante, la diferencia en capacidad predictiva no parece significativa por lo que, por su mayor confiabilidad, proponemos para Venezuela el uso de las escalas derivadas a partir de los análisis factoriales de los datos venezolanos. En cuanto a la capacidad predictiva de los factores de segundo orden, es muy pequeña, por lo que no parece aconsejable utilizarlas con fines aplicados. 
Tabla 6: Validez predicitiva

\begin{tabular}{|lc|cc|}
\hline \multicolumn{4}{|c|}{$\begin{array}{c}\text { Cuestionario CMC (Estructura española) } \\
\text { Predictores: Escalas de Primer Orden }\end{array}$} \\
Criterio: Nota en la asignatura en relación con la que se ha \\
completado el cuestionario CMC.
\end{tabular}

Tabla 7: Validez predicitiva

\begin{tabular}{|c|c|c|c|}
\hline \multicolumn{4}{|c|}{$\begin{array}{c}\text { Cuestionario CMC (Estructura venezolana) } \\
\text { Predictores: Escalas de Primer Orden } \\
\text { Criterio: Nota en la asignatura en relación con la que se ha } \\
\text { completado el cuestionario CMC. }\end{array}$} \\
\hline $\mathrm{R}=0.38$ & Probabilidad $=0.00$ & Sujetos: 420 & \\
\hline Predictores & $\begin{array}{c}\text { Peso con } \\
\text { Puntuaciones } \\
\text { directas }\end{array}$ & $\begin{array}{c}\text { Peso con } \\
\text { Puntuaciones } \\
\text { típicas }\end{array}$ & Probalidad \\
\hline Constante & 15.50 & 0.01 & 0.88 \\
\hline $\mathrm{F} 1$ & 0.00 & -0.44 & 0.00 \\
\hline $\mathrm{F} 2$ & -0.08 & 0.14 & 0.07 \\
\hline F3 & 0.03 & -0.12 & 0.05 \\
\hline $\mathrm{F} 4$ & -0.04 & 0.05 & 0.34 \\
\hline F5 & 0.02 & -0.03 & 0.63 \\
\hline F6 & 0.01 & & \\
\hline
\end{tabular}




\section{APÉNDICE A}

\section{CUESTIONARIO DE CLIMA DE CLASE}

-CMC VENZ- (Ampliado)

- Del cuestionario:

- De la adaptación venezolana
Jesús Alonso Tapia y P. García Pelaez.

Jesús Alonso Tapia y Luisa Irureta Núñez.

\section{INSTRUCCIONES}

Este cuestionario contiene una serie de afirmaciones que se refieren a cómo percibes el ambiente de tu clase, a lo que crees que valoran tus compañeros y profesores y a cómo trabajan habitualmente. Indica, pensando lo que ocurre en las clases de la asignatura por la que se te pregunta, el grado en que estás de acuerdo con cada afirmación.

Para responder, después de localizar en la hoja de respuestas el número correspondiente a la pregunta que vas a contestar, debes mostrar tu grado de acuerdo o desacuerdo tachando la alternativa deseada, teniendo en cuenta que cada número significa lo siguiente:

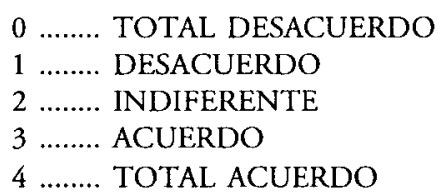

Si, por ejemplo, se te está preguntando en relación con las clases de Matemáticas y quisieras responder que estas totalmente de acuerdo con alguna de las afirmaciones, podrías contestar así:

$$
\text { X. } \begin{array}{lllll}
0 & 1 & 2 & 3
\end{array}
$$

La respuesta anterior significaría que estás totalmente de acuerdo con el contenido de la pregunta a la que estás respondiendo cuando se refiere a las clases de Matemáticas.

* El ObJETIVO de ESTA PRUEBA ES QUe nOS AYUdES A CONOCERTE MEJOR, DICIÉNDONOS COMO VES TU CLASE,

CON EL FIN DE PODER AYUDAR A MEJORAR LO QUE SEA POSIBLE.

* SÉ sincero EN TUS RESPUESTAS.

* NO DEJES NINGUNA PREGUNTA SIN CONTESTAR.

* NO ESCRIBAS NADA EN ESTE CUADERNILlO

* ESCRIBE SOlo EN LA hOJA de RESPUESTAS

GRACIAS POR TU COLABORACIÓN

Puedes empezar 
1. El profesor de esta materia explica con claridad cómo hay que hacer los trabajos.

* 2. Este profesor nos manda de un día para otro demasiadas tareas para hacer en casa.

3. El profesor explica los temas de tal forma que la mayoría (incluido yo) los comprendemos sin dificultad.

4. En esta materia es fácil atender al profesor o estudiar, porque casi ningún compañero interrumpe, molesta o alborota.

5. Este profesor hace más caso a los mejores alumnos.

6. En mi clase la mayoría de los alumnos prefiere trabajar en grupo.

7. En esta materia el profesor especifica cuáles son los puntos más importantes de cada tema.

8. Cuando tenemos que hacer un trabajo en clase, este profesor nos da poco tiempo para realizarlo.

9. No se necesita ser ningún genio para hacer los trabajos que nos manda el profesor, son fáciles.

10. En esta materia hay muchos ratos en los que no tenemos nada que hacer.

11. El profesor responde más a las preguntas de los mejores alumnos que a las de los menos buenos.

12. En mi clase, casi todos preferimos trabajar individualmente.

13. El profesor de esta materia se preocupa por enseñarnos cómo usar en la vida diaria lo que aprendemos en clase.

* 14. En general, en esta materia no nos sentimos agobiados ni por la cantidad de cosas que hay que hacer ni por la rapidez con que hay que hacerlas.

15. Considero que en esta materia, tal y como se enseña, la dificultad de la asignatura es grande.

16. En esta materia muchas veces el profesor tiene que dejar de explicar porque hay gente hablando o alborotando.

* 17. Este profesor, a la hora de poner las notas toma en cuenta lo que sabe cada uno, sin considerar lo que saben los demás.

18. En esta materia muchas veces trabajamos en grupo. 
Tabla 8: Validez predicitiva

\begin{tabular}{|lc|cc|}
\hline \multicolumn{4}{|c|}{$\begin{array}{c}\text { Cuestionario CMC (Estructura venezolana) } \\
\text { Predictores: Factores de Segundo Orden }\end{array}$} \\
Criterio: $\begin{array}{c}\text { Nota en la asignatura en relación con la que se ha } \\
\text { completado el cuestionario CMC. }\end{array}$ \\
\hline $\mathrm{R}=0.30$ & Probabilidad =0.00 & Sujetos: 420 \\
\hline Predictores & Peso con & $\begin{array}{c}\text { Peso con } \\
\text { Puntuaciones } \\
\text { típicas }\end{array}$ & Probalidad \\
\hline directas & & 0.00 \\
VCMC-I & 12.88 & -0.28 & 0.01 \\
VCMC-II & -0.01 & 0.13 & \\
\hline
\end{tabular}

\section{Referencias}

Alonso, J. (1992). Motivar en la adolescencia: Teoría, evaluación e intervención. Madrid, Servicio de Publicaciones UAM.

Alonso, J. y García; P. (1987). El cuestionario CMC. España. 2do. Congreso de Evaluación Psicológica.

Alonso, J. y Sola, J. (1987). Evaluación de la motivación de logro a través de los mensajes autodirigidos durante la realización de una tarea: el Cuestionario AM. Madrid. Trabajo no publicado. UAM.

Ames, C. y Ames. R. (Eds.) (1985 y 1984). Research on motivation in education Vols. 2 y 1. Nueva York: Academic Press.

Anderson, G. (1973). The assessment of learning environments: A manual for the learning environment inventory and the class inventory. Nueva Escocia: Atlantic Institute of Education.

Atkinson, .W. y Feather, N.T. (Eds.). (1966). A theory of achievement motivation. Nueva York, Wiley.

Dweck, C.S. (1986). Motivationsl processes affecting learning. American Psycologist, 41, 1040-1048.

Dweck, C.S. y Elliot, E.S. (1983). Achievment motivation. En E. M. Hetherington (Ed.): Socialization, personality and social development. New York: Wiley. 
Irureta, L. (En prensa). Querer aprender y saber pensar. Caracas: Monte Avila Editores - Fac. Humanidades y Educación. UCV

Irureta, L. (1995). Desarrollo de un programa de entrenamiento motivacional. Revista interamericana de Psicologia / Interamerican Journal of Psychology. 29. 1. 51-63.

Irureta, L. (1993). Cuándo y por qué los profesores motivan a sus estudiantes. Psicología. Revista de la Escuela de Psicología. Universidad Central de Venezuela. 28. 1-2. 51-61.

Irureta, L. (1993). Rendimiento académico. factores motivacionales de los alumnos. Psicologia. Revista de la Escuela de Psicología. Universidad Central de Venezuela. 28. 1-2. 51-61.

Irureta, L. (1990). Motivación de logro y aprendizaje escolar. Tesis Doctoral, Madrid, Universidad Autónoma.

Johnson, D.W. y Johnson, R. (1985). Motivational processes in cooperative, competitive, and individualistic learning situations. En C. Ames y R. Ames (Eds): Research on motivation in education (págs. 249-286). Orlando, Florida: Academic Press.

Owens, L. y Straton, G. (1980). The development of a cooperative, competitive and individualised learning preference scale for students. The Journal of Social Psychology, 50, 147-161.

Silbergeld, S., Koening, G.R. y Manderscheid, R.W. (1979). Classroom psychosocial environment. The Journal of Education Research, 69, 151155.

Silbergeld, S., Koening, G.R. y Manderscheid, R.W. (1977). Dimensions of classroom psychosocial environment. Journal of Comunity Psychology, 4, 299-306.

Weiner, B. (1986). An attributional theory of motivation and emotion. Nueva York: Springer-Verla 
19. Nuestro profesor hace que los trabajos que tenemos que hacer casi siempre nos resulten amenos e interesantes.

20. Este profesor explica muy rápido y da demasiada materia en cada clase.

21. En esta materia la mayoría de mis compañeros encuentran fáciles los exámenes que hace el profesor.

* 22. Considero que en esta materia, con demasiada frecuencia, hay quien se levanta y va de un lado a otro sin necesidad.

23. En esta materia se toma en cuenta, sobre todo, al que destaca por su inteligencia.

24. En mi clase los compañeros, en general, nos ayudamos unos a otros.

25. Este profesor nos manda trabajos, pero no nos explica qué objetivo persigue que logremos al hacerlos.

26. A nuestro profesor le gusta detenerse en cada tema el tiempo necesario para que lo entendamos.

27. Considero que el libro de texto de esta asignatura es bastante fácil de entender.

28. En esta materia no se pierde tiempo, siempre, tenemos algo que hacer.

* 29. En esta materia cada uno intenta hacer su trabajo lo mejor posible, sin preocuparse de si los demás lo hacen mejor o peor que él.

30. El profesor casi nunca nos compara, sobre todo se preocupa de decimos cómo mejorar lo que hemos hecho mal.

* 31. Cuando trabajamos en equipo lo que cuenta es el resultado final, porque el profesor nos evalúa a todo el grupo por igual.

32. El profesor no hace nada para que los trabajos que tenemos que hacer nos resulten más interesantes.

33. En esta materia los alumnos nos sentimos agobiados porque nos mandan a hacer muchas cosas y nos dan poco tiempo para terminarlas.

34. El profesor nos felicita individualmente cuando sacamos una nota mejor que otra que habiamos sacado antes.

35. Este profesor nos enseña de tal manera que la asignatura se nos hace fácil.

36. En esta materia el profesor nos trata a todos por igual, sin favoritismos. 
37. Al profesor no le preocupa la nota sino, sobre todo, lo que aprendemos.

38. Gracias al empeño del profesor, las clases empiezan con puntualidad.

39. Pienso, y creo que mis compañeros también, que trabajando en grupo se pierde mucho tiempo y se aprende menos que estudiando por separado.

40. En esta materia el profesor señala claramente qué objetivo tenemos que lograr al hacer cada trabajo.

41. El profesor pasa de un tema a otro rápidamente, pero ocurre con frecuencia que lo hace sin que hayamos entendido lo que nos ha explicado.

* 42. Cuando entrego un trabajo bien hecho al profesor, él lo evalúa positivamente sin darle demasiada importancia a los pequeños errores que pueda tener.

43. El profesor nos dice con frecuencia que lo importante es no perder de vista lo que uno se propone.

44. A los alumnos de esta materia nos resulta difícil entender cuando es el profesor quien explica los temas.

45. En esta materia da gusto estudiar: siempre sabemos lo que hay que hacer, nadie molesta y no se pierde tiempo.

* 46. Este profesor nunca nos enseña para qué puede servirnos en la vida diaria lo que aprendemos en clase.

47. Considero, como casi todos mis compañeros, que trabajando en grupo aprendemos más y mejor que trabajando solos.

* 48. El profesor casi siempre lee en voz alta las notas, así todos sabemos las notas de los demás.

49. En esta materia está muy claro quienes son los más inteligentes y los más torpes.

50. En mi clase cada quien se ocupa de lo suyo, y nadie te ayuda si lo necesitas.

51. Nuestro profesor habla con tranquilidad y sin apresuramiento, y no nos da demasiada materia cada día.

52. Por lo general, en esta materia no entendemos el libro de texto que tenemos este año.

* 53. El profesor no nos indica las partes de cada tema a las que tenemos que prestar más atención. 
54. En esta materia parece que nunca llega la hora de empezar a trabajar, la puntualidad brilla por su ausencia.

55. Cuando sacamos una buena nota el profesor nos felicita individualmente.

56. El profesor responde menos a las preguntas de los peores alumnos que a las de los mejores.

* 57. En esta materia da igual trabajar en grupo o no, porque el profesor siempre evalúa individualmente.

58. Con frecuencia el profesor nos manda a hacer trabajos, pero no nos explica cómo hay que hacerlos.

59. En mi clase, la nota que te pone el profesor no depende sólo de lo que tu sabes, sino también de lo que saben los demás.

* 60. La mayoría de mis compañeros quieren que su trabajo sea mejor que el de los demás.

61. En general durante las clases no nos levantamos de nuestros asientos, y cuando lo hacemos es por un motivo justificado.

62. En esta materia no hay quien estudie, se pierde mucho tiempo, hay demasiado alboroto y con frecuencia no sabemos que hacer.

63. Se necesita estudiar muchísimo para aprobar los exámenes que pone este profesor, considero que son realmente difíciles.

* 64. Este profesor nos manda pocas tareas para hacer en casa.

65. Con este profesor casi nunca trabajamos en grupo.

66. En mi clase el profesor valora a los alumnos sobre todo por las notas que sacan, más que por lo que aprenden.

67. Cuando el profesor nos manda a hacer un ejercicio en clase, nos da suficiente tiempo para que podamos terminarlo.

68. En esta materia el profesor hace más caso a los más inteligentes.

69. Este profesor se preocupa de que cada uno en particular aprenda.

70. En esta materia casi todos los compañeros encontramos demasiado dificiles los trabajos que tenemos que hacer.

71. Este profesor al explicar sigue el ritmo de los mejores, sin importarle que haya otros que no puedan seguirlo. 
72. Con este profesor frecuentemente trabajamos en grupo.

73. A este profesor le trae sin cuidado si aprendemos o no: él da sus clases y nada más.

74. En esta clase la mayoría pensamos que es mejor trabajar individualmente.

75. Generalmente este profesor consigue que en lugar de estar pensando siempre en ganar, colaboremos unos con otros.

76. El ritmo con que nuestro profesor explica permite que todos le sigamos y no sólo los mejores.

77. Este profesor, cuando nos asigna un trabajo, siempre trata de que nos quede bien claro qué es lo que pretende que aprendamos.

78. Este profesor le da casi más importancia a las notas que sacamos que a lo que aprendemos.

79. En esta clase la mayoría pensamos que trabajar en grupo no aporta ningún beneficio.

80. En esta asignatura da gusto: cuando no sabes algo siempre encuentras a alguien que te lo explique o te da pistas para entenderlo.

81. En esta materia, cuando estamos haciendo un trabajo en grupo, el profesor le dedica más tiempo a los grupos donde están los más inteligentes.

82. En esta asignatura cada quien hace los trabajos como mejor le parece, porque la mayoría de las veces no nos queda claro qué es lo que debemos hacer, y menos aún, cómo.

83. Este profesor lo que valora es el progreso que ha tenido cada uno con respecto a su rendimiento anterior y no si es mejor o peor que los demás.

84. En esta clase a la mayoría no nos gusta trabajar en grupo.

85. En esta materia cada uno tiene que resolver los problemas que encuentra: nadie te ayuda.

86. Cuando estamos haciendo un trabajo en grupo, este profesor atiende a todos los grupos por igual, sin importarle sin están los mejores o los peores alumnos.

87. En esta asignatura hay muchos compañeros que hacen bulla y no dejan atender.

88. Después de las evaluaciones, el profesor generalmente nos indica cuales fueron nuestros errores. 
* 89. En esta clase a la mayoría no nos gusta trabajar individualmente.

90. En esta asignatura por lo general trabajamos individualmente: el profesor dice que así cada quien demuestra lo que sabe.

91. Cuando hay que asistir o hacer alguna actividad especial, nuestro profesor siempre elige entre los más inteligentes.

92. En esta asignatura aprendemos poco porque, con frecuencia, no nos queda claro cómo tenemos que hacer las cosas.

93. A este profesor lo que le importa es el progreso personal de cada uno y no si sabe más o menos que los demás.

* 94. Cuando llevo un trabajo bien hecho al profesor, al evaluarlo le da demasiada importancia a los pequeños errores que pueda tener.

95. En esta asignatura por lo general trabajamos individualmente: el profesor dice que así no nos interferimos unos a otros y los mejores terminarán primero.

96. Nuestro profesor trata de que todos participemos en las actividades especiales de la materia y no que lo hagan sólo los mejores.

97. En esta materia generalmente no nos queda claro para qué hacemos los trabajos.

98. El profesor generalmente nos indica cuáles son las partes de cada tema a las que tenemos que prestar más atención.

99. En esta asignatura con frecuencia hacemos competencias: así podemos demostrar quienes son los mejores.

100 Este profesor se dedica a su grupo de preferidos, que generalmente son los más inteligentes, sin importarle lo que hacen los demás.

101 En las clases de csta materia generalmente se trabaja en grupo y ordenadamente.

102 En esta materia cada uno quiere ser el mejor y nos esforzamos para lograrlo.

103 Generalmente este profesor atiende a todos los estudiantes, pero le dedica más tiempo a quien lo necesita y no sólo a los mejores.

104 En esta asignatura casi siempre tenemos claro que objetivos se persiguen con las actividades que realizamos.

* 105 Con este profesor no sólo es importante aprender, sino también estar entre los mejores. 
$106 \mathrm{El}$ profesor de esta materia responde a cualquier pregunta: no le importa si quien la hace es de los mejores o de los peores alumnos.

107 En esta clase todos somos iguales: el profesor no tiene preferidos.

* No saturan en ningún factor

Del 1 al 70 corresponden a los elememtos del cuestionario original debidadmente adaptados.

Del 71 al 107 son las elementos diseñados especialmente para la versión venezolana. 
APENDICE B: Forma de obtener las puntuaciones en cada escala.

\begin{tabular}{|c|}
\hline ELEMENTOS QUE INTEGRAN CADA UNA DE LAS ESCALAS \\
\hline ESCALAS CORRESPONDIENTES A LOS FACTORES DE PRIMER ORDEN \\
\hline $\begin{aligned} \mathrm{F} 1= & \mathrm{CMC} 1+\mathrm{CMC} 7+\mathrm{CMC} 13+\mathrm{CMC} 19+\mathrm{CMC} 26+\mathrm{CMC} 30+\mathrm{CMC} 34 \\
& +\mathrm{CMC} 35+\mathrm{CMC} 37+\mathrm{CMC} 38+\mathrm{CMC} 40+\mathrm{CMC} 43+\mathrm{CMC} 55+ \\
& \mathrm{CMC} 69+(4-\mathrm{CMC} 73)+\mathrm{CMC} 75+\mathrm{CMC} 76+\mathrm{CMC} 77+\mathrm{CMC} 80 \\
& +\mathrm{CMC} 83+\mathrm{CMC} 86+\mathrm{CMC} 88+\mathrm{CMC} 93+\mathrm{CMC} 96+\mathrm{CMC} 98+ \\
& \mathrm{CMC102}+\mathrm{CMC} 103+\mathrm{CMC104}+\mathrm{CMC} 106\end{aligned}$ \\
\hline $\begin{aligned} \mathrm{F} 2= & (4-\mathrm{CMC} 3)+\mathrm{CMC} 8+(4-\mathrm{CMC} 9)+\mathrm{CMC} 15+\mathrm{CMC} 20+(4- \\
& \mathrm{CMC} 21)+\mathrm{CMC} 25+(4-\mathrm{CMC} 27)+\mathrm{CMC} 32+\mathrm{CMC} 33+\mathrm{CMC} 41 \\
& +\mathrm{CMC} 44+(4-\mathrm{CMC} 51)+\mathrm{CMC} 52+\mathrm{CMC} 58+\mathrm{CMC} 63+(4- \\
& \mathrm{CMC} 67)+\mathrm{CMC} 70+\mathrm{CMC} 82+\mathrm{CMC} 2+\mathrm{CMC} 97\end{aligned}$ \\
\hline $\begin{aligned} \mathrm{F} 3= & \mathrm{CMC} 5+\mathrm{CMC} 11+\mathrm{CMC} 23+(4-\mathrm{CMC} 36)+\mathrm{CMC} 49+\mathrm{CMC} 56 \\
& +\mathrm{CMC} 66+\mathrm{CMC} 68+\mathrm{CMC} 71+\mathrm{CMC} 78+\mathrm{CMC} 81+\mathrm{CMC} 91+ \\
& \mathrm{CMC} 99+\mathrm{CMC} 100+(4-\mathrm{CMC} 107)\end{aligned}$ \\
\hline $\begin{aligned} \mathrm{F} 4= & (4-\mathrm{CMC} 6)+\mathrm{CMC12}+(4-\mathrm{CMC} 24)+\mathrm{CMC} 39+(4-\mathrm{CMC} 47) \\
& +\mathrm{CMC} 50+\mathrm{CMC74}+\mathrm{CMC} 79+\mathrm{CMC} 4+\mathrm{CMC} 85+\mathrm{CMC} 95\end{aligned}$ \\
\hline $\begin{aligned} \mathrm{F} 5= & (1-\mathrm{CMC} 4)+\mathrm{CMC10}+\mathrm{CMC16}+\mathrm{CMC} 22+(4-\mathrm{CMC} 28)+(4 \\
& -\mathrm{CMC} 45)+(4-\mathrm{CMC} 61)+(4-\mathrm{CMC} 62)+\mathrm{CMC} 87\end{aligned}$ \\
\hline $\begin{aligned} \mathrm{F} 6= & \mathrm{CMC18}+\mathrm{CMC54}+\mathrm{CMC59}+(4-\mathrm{CMC} 65)+\mathrm{CMC72}+(4- \\
& \mathrm{CMC} 90)+\mathrm{CMC} 101\end{aligned}$ \\
\hline ESCALAS CORRESPONDIENTES A LOS FACTORES DE SEGUNDO ORDEN \\
\hline VCMC-I $=(148-F 1)+F 2+F 3+F 5$ \\
\hline $\mathrm{VCMC}-\mathrm{II}=(60-\mathrm{F} 4)+\mathrm{F} 6$ \\
\hline
\end{tabular}

